\title{
Is there a Correlation between Systolic Heart Failure and Levels of Toll-Like Receptor-5 and N-Terminal Pro-B-Type Natriuretic Peptide?
}

\author{
Cetin Mirzaoglu, Tarik Kivrak ${ }^{1}$, Mehmet Balin ${ }^{1}$, Mehmet Ali Kobat' ${ }^{1}$, Orhan Dogdu ${ }^{2}$, Ilgin Karaca ${ }^{1}$ \\ Department of Cardiology, Elazig State Hospital, 'Department of Cardiology, Firat University Hospital, ${ }^{2}$ Department of Cardiology, Elazig Medical Park Hospital, Elazig, \\ Turkey
}

\section{Abstract}

Objective: Specific biomarkers are essential in the diagnosis of heart failure. Our trial aim is determined that relationship between toll-like receptor-5 (TLR-5) and N-terminal pro-B-type natriuretic peptide (NT-ProBNP) in patients with reduced ejection fraction. Methods: Two groups were formed in our study (normal and patient group). Among the two groups were investigated that relationship between TLR-5 and NT-ProBNP. Results: The plasma levels of both NT-ProBNP and TLR-5 are significantly higher in patients with congestive heart failure than healthy individuals. However, there is no definite correlation between plasma levels of NT-ProBNP and TLR-5 in patients with congestive heart failure. The high-level plasma TLR-5 is of prognostic value independent from the plasma NT-ProBNP levels, in these patients. Conclusion: As a conclusion, according to recent studies, the high plasma levels of NT-ProBNP and TLR-5 are mainly associated with high mortality and longer hospitalization rate in individuals with heart failure. Therefore, the higher is plasma levels of markers such as TLR-5 and NT-ProBNP, the worse is the overall prognosis in these patients. NT- ProBNP and TLR-5 are thought to be the cheapest and the most appropriate marker to be determined.

Keywords: N-terminal pro-B-type natriuretic peptide, systolic heart failure, toll-like receptor

\section{INTRODUCTION}

Heart failure is a syndrome that occurs as a result of a decline in the ability of the heart to send enough blood to the tissues to meet the changing oxygen and metabolic needs of the body. Today, the clinic and echocardiographic findings of the patients have an essential role in the diagnosis of heart failure. However, new generation biochemical markers of cardiac ventricle origin may give important clues for the determination of heart failure, and further studies on finding newer cardiac markers are continuing worldwide. Many biochemical parameters are used in the diagnosis of heart failure. ${ }^{[1]}$ The heart muscle is also an endocrine system besides its blood-pumping task. In this context, atrial natriuretic peptide is secreted from the atrium and brain natriuretic peptide (BNP) is emitted from the ventricle in response to the tension of myocyte cells in the atrium and ventricle walls of the heart. N-terminal pro-B-type natriuretic peptide (NT-ProBNP) is today meaningful for the preliminary diagnosis of heart failure. NT-ProBNP type

\begin{tabular}{|l|l|}
\hline \multicolumn{2}{|c|}{ Access this article online } \\
\hline Quick Response Code: & Website: \\
\hline & http:/www.ijcva.com \\
\hline & \\
\hline
\end{tabular}

natriuretic peptide is a diuretic peptide synthesized in the ventricular myocardium but released into the plasma only in response to the left heart failure conditions such as the increase in the intraventricular volume, wall tension, and end diastolic pressure. Its specificity and sensitivity for diagnosing heart failure were published about $95 \%$ in the 2001 Practical Guideline of American College of Cardiology and American Heart Association. It is a valuable non-invasive method even for the heart failure cases who are still in the asymptomatic phase. It is also known that heart failure has inflammatory processes. Toll-like receptor-5 (TLR-5) as an inflammatory marker can give us illuminating information about this disease. The most commonly used method of diagnosis is

Address for correspondence: Dr. Tarik Kivrak, Department of Cardiology, Firat University Hospital, Elazig, Turkey. E-mail: tarikkivrak@gmail.com

This is an open access journal, and articles are distributed under the terms of the Creative Commons Attribution-NonCommercial-ShareAlike 4.0 License, which allows others to remix, tweak, and build upon the work non-commercially, as long as appropriate credit is given and the new creations are licensed under the identical terms.

For reprints contact: reprints@medknow.com

How to cite this article: Mirzaoglu C, Kivrak T, Balin M, Kobat MA, Dogdu O, Karaca I. Is there a correlation between systolic heart failure and levels of toll-like receptor-5 and N-terminal pro-B-type natriuretic peptide? Int J Cardiovasc Acad 2018;4:65-9. 
echocardiography (ECG). However, the need for experienced physicians, the cost and duration of ECG may be found less advantageous compared to biochemical parameters. Plasma NT-ProBNP and TLR-5 measurements seem more beneficial than ECG because they are inexpensive, easy to measure, measurable in a short time and easy to evaluate. In this study; we measured plasma NT-ProBNP and TLR-5 levels in patients who are diagnosed with clinical and echocardiographic heart failure. We investigated if the role of echocardiographic findings and these two biochemical markers are concordant in the diagnosis of heart failure. Hence in this study, comparison of two different methods in the diagnosis of heart failure is the issue. We tried to determine the superiority of these two approaches to diagnose heart failure; TLR-5 and NT-ProBNP method which is cheaper and more accessible, and ECG method which is expensive, time-consuming and requires the experienced physician.

\section{Methods}

Patients admitted to the Cardiology outpatient clinic of the Firat University Research Hospital between May 2015, and May 2016 prospectively included in the study. We added in 17 female and 33 male to our research, totally 50 patients that we diagnosed heart failure and 25 males and 25 females, totally 50 healthy people as the control group was enrolled in the study. Ethics committee approval received from the Local Committee. Some of the patients who were admitted to our cardiology outpatient clinic with dyspnea in the effort, shortness of breath at night, tiredness, fatigue, anorexia, coughing, nausea, a manifestation of jugular veins, swelling in feet and legs, were diagnosed heart failure after a physical examination and clinical evaluations. ECG, chest radiography and ECG were performed to these patients. Blood samples were collected from heart failure patients with ejection fraction (EF) $\leq 30 \%$ in ECG; plasma NT-ProBNP, TLR-5, C-reactive protein (CRP), routine biochemical tests, and hemogram, sedimentation rate were studied. In the control group and all patients, detailed anamnesis was obtained, and physical examinations were performed. Patients with the chronic inflammatory disease, active infection, advanced liver disease, severe renal disease (creatinine $\geq 2.5$ ), advanced heart valve disease, pulmonary artery pressure $>35$ and chronic obstructive pulmonary disease (COPD) were excluded from the study. Blood samples were taken after a 10-12 $\mathrm{h}$ between 08:00 and 10:00. The samples were kept at room temperature for $30 \mathrm{~min}$ and then centrifuged at $4000 \mathrm{rpm}$ (1600 g). BD vacutainer tubes were used for blood collection. Serum NT-ProBNP levels were studied by electrochemiluminescence assay using a modular Roche Hitachi E-70 analyzer (Roche Diagnostics, Germany). Routine biochemistry tests were performed spectrophotometrically on the Aerosat 2.0 (Abbott laboratory) instrument. In serum samples, TLR-5 was studied by using ELISA method following the directives of the manufacturer company (Hangzhou EastbioFarm Co. Ltd. Human TLR5 ELISA kit Catalog Number: CK-E90260).
All echocardiographic measurements were made in a left lateral decubitus position by a transthoracic approach using a General Electric Vivid PRO 7-color Doppler ECG device with a $2.5-3.5 \mathrm{MHz}$ transducer. M-mode recordings were made at rate $50 \mathrm{~mm} / \mathrm{s}$, and Doppler recordings were made at rate $100 \mathrm{~mm} / \mathrm{s}$. M-mode and 2-D measurements were performed according to the recommendations of the American Society of ECG from parasternal long axis view. Statistical analysis of the study was made using the Statistical Package for Social Sciences 21 (Chicago, IL, USA) statistical program. Characteristics of the patients were summarized with basic statistics. Arithmetic means standard deviation were used for numerical parameters; while numbers and percentage values were used for categorical variables. In the comparisons, the Kolmogorov-Smirnov test was used to determine the distribution of all variable groups. Parametric statistical methods were used for variables with normal distribution, and non-parametric statistical methods were used for variables with skewed distribution. Student $t$-test (Independent Sample $t$-test) was used as a parametric test, and the Mann-Whitney U-test was used as a nonparametric test. Cross-tabulation statistics were used to compare categorical variables (Chi-square). In all analyzes, the statistical significance limit was accepted as $P<0.05$.

\section{RESULTS}

Age of patients was $68( \pm 9)$ years old in our study and $27.9( \pm 9.3)$ years old in the control group. There was the statistically significant difference between the groups regarding HT, diabetes mellitus (DM), hyperlipidemia, and coronary artery disease history $(P<0.001)$. In the patient group, hypertension (\%74), DM (\%52), hyperlipidemia (\%58), and coronary artery disease $(\% 78)$ were found to be more frequent than the control group [Table 1]. When the mean hemogram and biochemistry values of patient and control

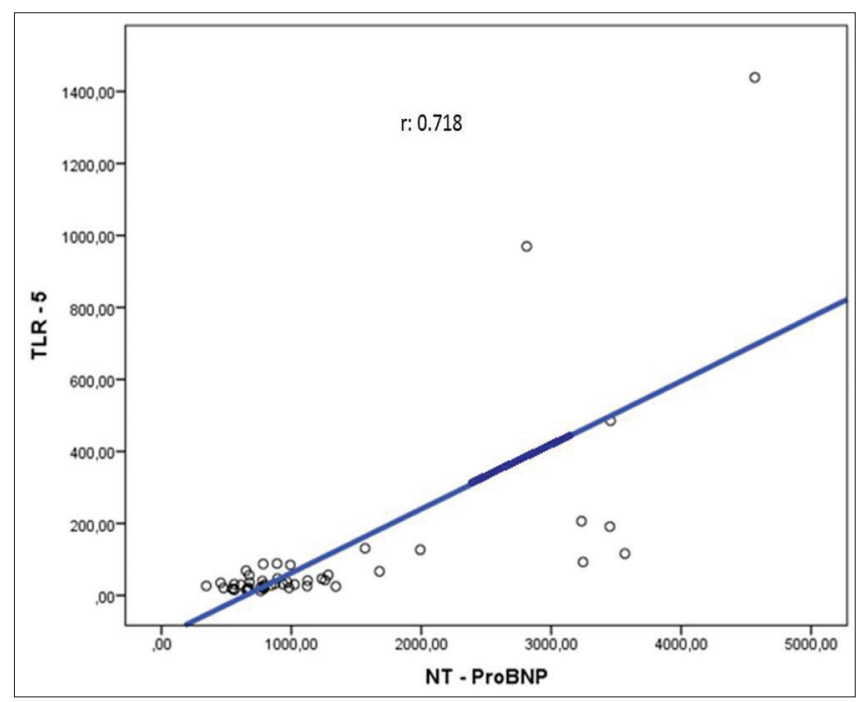

Figure 1: Relationship between TLR-5 and NT-ProBNP $(r=0.718$, $P<0.001)$ 
groups were compared, a significant difference was found regarding laboratory values in the patient's group [Table 2]. There was the statistically significant difference between the two groups regarding echocardiographic values. In the patient group, many benefits were found to be higher; EF was found to be lower [Table 3]. In patients group, we determined that TLR and NT-ProBNP values are high [Table 4]. There was a positive correlation between TLR and NT-ProBNP $(r=0.718, P<0.001)$ [Figure 1].

\begin{tabular}{lccc}
\hline \multicolumn{4}{c}{ Table 1: Basic demographic features } \\
\\
$\begin{array}{l}\text { Patient Group } \\
(\boldsymbol{n}=\mathbf{5 0})\end{array}$ & $\begin{array}{c}\text { Control Group } \\
(\boldsymbol{n}=\mathbf{5 0})\end{array}$ & $\boldsymbol{P}$ \\
\hline Age & $68 \pm 9$ & $27,9 \pm 9,3$ & $<0,001$ \\
Gender (Male) & 33 & 25 & 0,06 \\
Hypertension & $\% 74$ & $\% 4$ & $<0,001$ \\
Diabetes Mellitus & $\% 52$ & $\% 1$ & $<0,001$ \\
Hyperlipidemi & $\% 58$ & $\% 4$ & $<0,001$ \\
Smoking & $\% 12$ & $\% 26$ & 0,07 \\
Coronary artery disease (\%) & $\% 78$ & $\% 2$ & $<0,001$ \\
\hline
\end{tabular}

\begin{tabular}{lccc}
\hline Table 2: Basic laboratory values & & \\
\hline & $\begin{array}{c}\text { Patient Group } \\
(\boldsymbol{n}=\mathbf{5 0})\end{array}$ & $\begin{array}{c}\text { Control Group } \\
(\boldsymbol{n}=\mathbf{5 0})\end{array}$ & $\boldsymbol{P}$ \\
\hline Hemoglobin & $12,6 \pm 1,8$ & $14,1 \pm 1,3$ & $<0,001$ \\
White Blood Cell & $8,9 \pm 3$ & $7,1 \pm 1,6$ & $<0,001$ \\
Platellet & $244,9 \pm 8,88$ & $270,5 \pm 58,5$ & 0,07 \\
Sedimentation & $29,1 \pm 12,5$ & $6,8 \pm 3,7$ & $<0,001$ \\
CRP & $16,4 \pm 7,9$ & $3,6 \pm 1,2$ & $<0,001$ \\
Sodium & $137,8 \pm 4,5$ & $141,1 \pm 2,3$ & $<0,001$ \\
Potassium & $4,27 \pm 0,76$ & $4,39 \pm 0,35$ & 0,34 \\
Urea & $63,6 \pm 32,5$ & $27,8 \pm 7,3$ & $<0,001$ \\
Creatinine & $1,45 \pm 1,1$ & $0,7 \pm 0,15$ & 0,003 \\
\hline
\end{tabular}

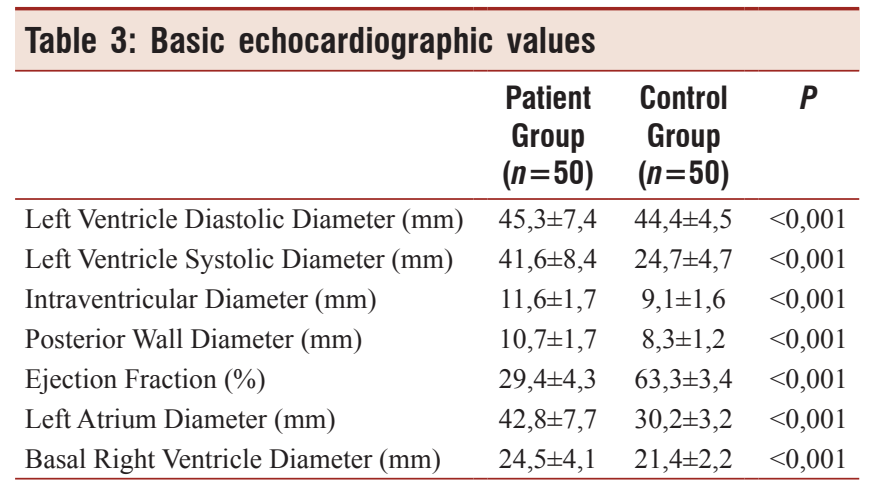

Table 4: Group difference between TLR-5 and NT-ProBNP

\begin{tabular}{lccc}
\hline & $\begin{array}{c}\text { Patient Group } \\
(\boldsymbol{n}=\mathbf{5 0 )}\end{array}$ & $\begin{array}{c}\text { Control Group } \\
(\boldsymbol{n}=\mathbf{5 0 )}\end{array}$ & $\boldsymbol{P}$ \\
\hline Toll Like Receptor-5 & $86,5 \pm 62,2$ & $45,3 \pm 19,9$ & 0,003 \\
NT-ProBNP & $1274 \pm 996$ & $106 \pm 65$ & 0,001 \\
\hline
\end{tabular}

\section{Discussion}

Heart failure is an increasing health problem which has high morbidity and mortality. ${ }^{[1]}$ The incidence of heart failure does not decrease in spite of progressive and successful developments in the treatment of etiologic factors such as ischemic heart disease and hypertension.

On the contrary, it is an increasingly common and significant public health problem. ${ }^{[2]}$ The increase in the proportion of older adults in the community and developments of treatment of myocardial infarction have a considerable influence on this situation. As the diagnostic and therapeutic possibilities of myocardial infarction increase, the number of surviving patients increases and some of these people become chronic heart failure patients. ${ }^{[2]}$ Despite the relatively younger population in our country, when it is considered that the average lifespan is prolonged and rate of cardiovascular diseases is increased, it seems that heart failure is a severe problem that needs to be addressed.

Since there are no symptoms specific to heart failure, to reduce morbidity and mortality, it becomes more important to diagnose early and start treatment soon for a differential diagnosis. Shortness of breath complaint can be seen in many diseases, especially COPD, and causes difficulties about distinguishing the disease that causes the primary charge in the patients, especially who have both heart failure and COPD diseases in the emergency department. ${ }^{[2]}$ Here, the delay in diagnosis and treatment contributes to the morbidity and mortality of the patient.

Heart failure is a progressive disease, and besides, it is difficult to predict which patients have a high risk of death and cardiovascular event. Many parameters that are shown to be indicative of mortality, such as New York Heart Association (NYHA) classification, advanced age, and DM. ${ }^{[2]}$ For this reason, there is a need for parameters that can show prognosis and patients with high mortality risk better. Similarly, starting an effective treatment to these patients in the early period and monitoring the effectiveness of the procedure is as important as determining the prognosis.

NT-ProBNP, which first appeared years ago but has been recently recognized as cardiologically important, is now meaningful for the preliminary diagnosis of heart failure. NT-ProB type natriuretic peptide (NT-ProBNP) is a diuretic peptide synthesized in the ventricular myocardium and released into the plasma in response to left heart failure conditions such as the increase in ventricular volume, wall tension, and end diastolic pressure. In the European Society of Cardiology guideline, it is published that the specificity and sensitivities of NT-proBNP for diagnosing heart failure were about $95 \%$. It is a valuable non-invasive method even for identification of yet asymptomatic heart failure cases. In previous studies, Doust et al., ${ }^{[3-5]}$ Maisel $^{[6]}$ and Remme and Swedberg ${ }^{[7,8]}$ have shown that NT-ProBNP can be used to diagnose symptomatic heart failure. Yoshimura et al., ${ }^{\left[{ }^{[]}\right.}$Wieczorek et al., ${ }^{[10]}$ and Maisel 
et $a l .{ }^{[11]}$ have shown that NT-ProBNP is correlated with the NYHA stage in their studies. The findings we obtained in our study were consistent with the results obtained in previous studies.

Regarding comparing NT-proBNP level with ECG, the survey of Kaan et al. is also essential and simultaneous plasma. NT-proBNP levels were assessed in the cases to whom ECG performed to determine left the ventricular function. ${ }^{[12]} \mathrm{Left}$ ventricular dysfunction was detected in half of the facts in the group without known heart failure and previous left ventricular dysfunction, and plasma NT-proBNP levels were significantly higher in these cases. Abnormal echocardiographic findings were found in all patients in the group with known heart failure or previous left ventricular dysfunction, and the NT-proBNP levels of this group were found to be even higher. In addition to patients with heart failure with systolic dysfunction, plasma NT-proBNP levels also increase in patients with heart failure with diastolic dysfunction. In our study, consistent with the literature, NT-ProBNP values of the cases with $\mathrm{EF}<30 \%$ were statistically significantly higher than those with $\mathrm{EF}>50 \%(P<0.001)$, and there was a weak negative correlation between NT-ProBNP values and EF\% $(r=-0.360 P<0.001)$.

TLRs are a group of type 1 transmembrane proteins that provide the natural immune response against many pathogens. It was named "toll" because of its similarity to the receptor "toll gene," which was first discovered in 1991 in Drosophila melanogaster and believed to have an important function in the immune system response. Today, these molecules which are homologous to the interleukin-1 receptor in humans are called "TLR." These receptors are found in mast cells, dendritic cells, eosinophils, neutrophils, natural killer cells, natural killer T cells and particularly in macrophages; and they play a role in recognition of microorganisms and triggering the inflammation. Until now, 10 TLRs in the human and the bovine and 13 TLRs in the mouse have been identified, each of which can bind to one or more specific microbial molecules. 13 members have been recognized in the TLR family. Ligands of TLR1-TLR9 and TLR11 were determined; but the ligands of TLR10, TLR12, and TLR13 are not yet known. TLR5 recognizes bacterial flagellin, an essential structural component of bacterial flagella. The binding of TLR 5 to flagellin results in an inflammatory signal as tumor necrosis factor- $\alpha$. Therefore, that can be used as an inflammatory marker. It is known that other inflammatory markers (hs-CRP, N/L ratio) are high in systolic heart failure. TLR 5, as a new inflammatory biomarker, may also be a marker of decompensated systolic heart failure.

In our study, the values of both TLR-5 and NT-ProBNP were statistically significantly higher in patients with systolic heart failure $(\mathrm{EF}<30 \%)$ than in those without heart failure $(\mathrm{EF}>55 \%)(P<0.001)$. Besides, consistent with the literature, there was no significant correlation of NT-ProBNP and TLR-5 values between cases with heart failure and those without heart failure $(P>0.05)$. These results show that NT-ProBNP and TLR-5 levels are significantly higher in heart failure patients but, consistent with the literature, prognostic value of high TLR-5 is found to be a separate risk for heart failure independent of NT-ProBNP. Furthermore, there is a poor positive correlation between age and NT-ProBNP and TLR-5 values in our study. $R=0.246 P<0.05$ and $r=0.223$ $P<0.05$ (NT-ProBNP and TLR-5 values increase with increasing age). There is a poor positive correlation between urea and NT-ProBNP and TLR-5 values $(r=0.409 P<0.001$ and $r=0.208 P<0.01)$. There is no significant correlation between fasting blood sugar, alanine aminotransferase, aspartate transaminase, gamma-glutamyl transferase, $\mathrm{K}, \mathrm{CL}$ values and NT-ProBNP and TLR-5 levels. There was no statistically significant difference in NT-ProBNP and TLR-5 values between patients with and without arrhythmia or between the patients with and without wide QRS in ECG $(P<0.05)$.

\section{ConcLusion}

Pro-BNP and TLR-5 levels were found to be significantly higher in patients with systolic heart failure than in those without heart failure. The high level of TLR-5, like NT pro-BNP, may be associated with mortality and number and duration of hospitalization. The routine use of this biomarker in systolic heart failure requires a large number of further studies involving more patients.

\section{Financial support and sponsorship}

Nil.

\section{Conflicts of interest}

There are no conflicts of interest.

\section{References}

1. Ponikowski P, Voors AA, Anker SD, Bueno H, Cleland JG, Coats AJ, et al. 2016 ESC guidelines for the diagnosis and treatment of acute and chronic heart failure: The task force for the diagnosis and treatment of acute and chronic heart failure of the European Society of Cardiology (ESC). Developed with the special contribution of the heart failure association (HFA) of the ESC. Eur J Heart Fail 2016;18:891-975.

2. McDonagh TA, Gardner RS, Clark AL, Dargie HJ. Oxford Textbook of Heart Failure. Oxford University Press; 2011. ISBN: 9780199577729.

3. Vanderheyden M, Bartunek J, Goethals M. Brain and other natriuretic peptides: Molecular aspects. Eur J Heart Fail 2004;6:261-8.

4. Vesely DL. Natriuretic peptides and acute renal failure. Am J Physiol Renal Physiol 2003;285:F167-77.

5. Doust JA, Glasziou PP, Pietrzak E, Dobson AJ. A systematic review of the diagnostic accuracy of natriuretic peptides for heart failure. Arch Intern Med 2004;164:1978-84.

6. Maisel A. B-type natriuretic peptide in the diagnosis and management of congestive heart failure. Cardiol Clin 2001;19:557-71.

7. Remme WJ, Swedberg K. Guidelines for heart evaluation and management of chronic heart failure. J Am Cardiol 1992;520:156-62.

8. Remme WJ, Swedberg K; Task Force for the Diagnosis and Treatment of Chronic Heart Failure, European Society of Cardiology. Guidelines for the diagnosis and treatment of chronic heart failure. Eur Heart $\mathrm{J}$ 2001;22:1527-60.

9. Yoshimura M, Yasue H, Okumura K, Ogawa H, Jougasaki M, Mukoyama $\mathrm{M}$, et al. Different secretion patterns of atrial natriuretic peptide and brain natriuretic peptide in patients with congestive heart 
failure. Circulation 1993;87:464-9.

10. Wieczorek SJ, Bailly KR, Thomas P. Clinical evaluation of the Triage B type natriuretic peptide assay for point of core testing of patients with congestive heart failure. Clin Chem 2000;46:77-8.

11. Maisel AS, Krishnaswamy P, Nowak RM, McCord J, Hollander JE,
Duc P, et al. Rapid measurement of B-type natriuretic peptide in the emergency diagnosis of heart failure. N Engl J Med 2002;347:161-7.

12. Kaan J, Hope J, Garcia A. A rapid bedside test for brain natriuretic peptide accurately predict cardiac function in patients referred for echocardiography. J Am Coll Cardiol 2000;135:419-20. 